\title{
Effect of Heparin on Serum Free-fatty-acids, Plasma Catecholamines, and the Incidence of Arrhythmias following Acute Myocardial Infarction
}

\author{
P. G. NELSON,* M.B., M.R.C.P.
}

\begin{abstract}
Cummary: The effect of intravenous heparin in a thera$\checkmark$ peutic dosage on cardiac arrhythmias in patients with indubitable acute myocardial infarction was investigated. The value of serum free-fatty-acids (F.F.A.s) and plasma catecholamines in the prediction of patients vulnerable to serious arrhythmias was also studied.

Heparin produced a significant rise in F.F.A., maximal within 10 minutes. of injection, but did not increase the incidence of cardiac arrhythmias.

No relationship was found between the incidence of arrhythmias and the initial levels of F.F.A. or adrenaline. No correlation was obtained between F.F.A. and plasma catecholamine levels. Heparin did not have a consistent effect on plasma catecholamines. Initial control plasma noradrenaline concentrations, however, were found to be significantly correlated with the incidence of subsequent arrhythmias. It is suggested that the level of plasma noradrenaline may be a valuable predictive guide to those patients likely to develop significant arrhythmias after acute myocardial infarction.
\end{abstract}

\section{Introduction}

High levels of serum free-fatty-acids (F.F.A.s) occur soon after the onset of acute myocardial infarction (Kurien and Oliver, 1966; Oliver et al., 1968). Maximal levels are attained within 36 hours. At this time high levels of plasma catecholamines have also been found (Gazes et al., 1959; Richardson, 1963). F.F.A.s have been related to the incidence of early serious arrhythmias following acute myocardial infarction (Oliver et al., 1968; Gupta et al., 1969). Plasma catecholamines have also been so related (McDonald et al., 1969). High plasma levels of catecholamines can cause mobilization of F.F.A. from adipose tissue with resultant rise in circulating F.F.A.s (Carlson, 1968). Both F.F.A. (Kurien et al., 1969) and plasma catecholamines (Staszewska-Barczak and Ceremużyński, 1968; Ceremużyński et al., 1969) have been shown to produce cardiac arrhythmias in the experimental animal with acute myocardial infarction. It has not been clear hitherto which is the more important factor in the causation of these arrhythmias.

It has been suggested that measurement of F.F.A. levels may be of value in the prediction of those patients liable to develop serious arrhythmias following myocardial infarction (Oliver et al., 1968). Other investigators have been unable to confirm this work (Rutenburg et al., 1969; Rutenburg and Soloff, 1970). Heparin has been used for many years for the prevention of thromboembolism complicating acute myocardial infarction. Heparin, however, is known to cause a rise in F.F.A.s, and the safety of its use in this clinical situation has recently been questioned critically (Kurien et al., 1969; Rutstein et al., 1969). A recent study has suggested that about one-third of the patients with acute myocardial infarction develop calf vein thrombosis (Maurer, 1970). Since heparin is an effective anticoagulant with rapidly reversible properties it was important to determine whether it initiated or aggravated cardiac arrhythmias in patients with acute myocardial infarction. * Research Fellow in Cardiology, Royal Victoria Hospital, Belfast, Northern
Ireland.

\section{Patients and Methods}

Twenty-four patients with a diagnosis of acute myocardial infarction were included in the trial. There were 20 men and 4 women (aged 37 to 73 years). The diagnosis was considered established when there was (a) indubitable- electrocardiographic evidence of recent infarction or sequential ST or T wave changes accompanied by significant and transient rise in serum aspartate aminotransferase, or (b) left bundle-branch block with similar enzyme changes. These patients werc among those admitted to the coronary care unit in this hospital between 1 August, 1969 and 1 January, 1970. They were accepted for study provided that the onset of symptoms had been less than 24 hours before admission (16 patients were admitted within six hours). None of the patients fell into the severe category of myocardial infarction-that is, cardiogenic shock and/or congestive heart failure. It was ascertained that none of them had received heparin, $\beta$-adrenergic blocking agents, antihypertensive drugs, clofibrate, or adrenergic drugs before admission. Electrocardiograms were continuously monitored on a television screen and also by oscilloscope in the patient's cubicle. The E.C.G. could be recorded permanently at any time, and memory tapes were available to replay what had happened during the preceding 40 seconds. Monitoring was continuous during the patient's stay in the coronary care unit.

On admission to the unit the patients were classified with respect to age, sex, time from onset of symptoms, and site and severity of infarct with a view to matching in pairs. The first patient in a potential pair was randomly allocated to one of two treatments, and the second patient, if a suitable match, was automatically allocated to the alternative treatment. Treatment consisted either of an intravenous injection of 12,500 units of heparin or $2.5 \mathrm{ml}$. of $5 \%$ dextrose intravenously. Venous blood samples were obtained for F.F.A. estimation before and 10,30, and 60 minutes after injection. Those patients receiving heparin had concurrent samples taken for catecholamine estimation, while those receiving dextrose had one catecholamine sample taken with the control F.F.A. sample. All blood samples were immediately centrifuged at $3,000 \mathrm{~g}$ for 15 minutes. F.F.A.s were extracted by using Trout's (1960) modification of Dole's technique and estimated by the photometric method of Mosinger (1965) (normal values: mean $854 \mu \mathrm{mole} / 1$., range $620-1,150 \mu \mathrm{mole} / 1$.). plasma catecholamines were determined with McCullough's (1968) modification of Merrill's method (normal values: noradrenaline-mean $0.74 \mu \mathrm{g} . / 1$., range $0.35-1.15 \mu \mathrm{g} . / 1$.; adrenaline-mean 0.27 $\mu \mathrm{g}$./1., range 0.1-0.4 $\mu \mathrm{g}$./1.).

A continuous E.C.G write-out was recorded during the 15 minutes before and the hour after injection. This was analysed for frequency of ventricular ectopic beats and incidence of other significant arrhythmias. Note was taken of any further arrhythmias occurring during the patient's stay in the coronary care unit. Significant arrhythmias were defined as :

Supraventricular: sinus bradycardia, five or more atrial ectopics per minute, supraventricular tachycardia, atrial flutter, atrial fibrillation, and nodal rhythm.

Ventricular: five or more ventricular ectopics per minute, consecutive ventricular ectopics, $R$ on $T$ ventricular ectopics, ventricular tachycardia, ventricular flutter, and ventricular fibrillation. 


\begin{tabular}{|c|c|c|c|c|c|c|c|c|c|c|c|c|c|c|c|c|c|c|c|c|c|}
\hline \multirow[b]{2}{*}{$\begin{array}{l}\text { Case } \\
\text { No. }\end{array}$} & \multirow[b]{2}{*}{$\begin{array}{l}\text { H } \\
\text { or } \\
\text { D }\end{array}$} & \multirow[b]{2}{*}{$\begin{array}{l}\text { Age } \\
\text { and } \\
\text { Sex }\end{array}$} & \multirow[b]{2}{*}{$\begin{array}{c}\text { Site } \\
\text { of } \\
\text { Infarct }\end{array}$} & \multirow[b]{2}{*}{$\begin{array}{c}\text { Severity } \\
\text { of } \\
\text { Infarct }\end{array}$} & \multirow{2}{*}{$\begin{array}{c}\text { Time } \\
\text { from } \\
\text { Onset of } \\
\text { Symptoms } \\
\text { (Hours) }\end{array}$} & \multicolumn{4}{|c|}{ F.F.A.s ( $\mu$ mole $/ 1)}$. & \multicolumn{4}{|c|}{ Noradrenaline (g./1.) } & \multicolumn{4}{|c|}{ Adrenaline (g./l.) } & \multicolumn{2}{|c|}{ No. of V.E.s } & \multirow{2}{*}{$\begin{array}{c}\text { Other } \\
\text { Arrhythmias } \\
\text { during } \\
\text { Post-iniection } \\
60 \text { mins. }\end{array}$} & \multirow{2}{*}{$\begin{array}{l}\text { Arrhythmins in } \\
\text { Coronary } \\
\text { Care Unit }\end{array}$} \\
\hline & & & & & & Control & $10 \mathrm{~min}$. & $30 \mathrm{~min}$. & $60 \mathrm{~min}$. & Control & $10 \mathrm{~min}$. & $30 \mathrm{~min}$. & $60 \mathrm{~min}$. & Control & $10 \mathrm{~min}$. & $30 \mathrm{~min}$. & $60 \mathrm{~min}$. & $\begin{array}{l}\text { Control } \\
15 \text { mins. }\end{array}$ & $\begin{array}{c}\text { Post- } \\
\text { inection } \\
60 \text { mins. }\end{array}$ & & \\
\hline$\frac{1}{2}$ & $\begin{array}{l}\mathrm{H} \\
\mathrm{D}\end{array}$ & $37 \mathrm{M}$. & $\hat{A}$ & $\begin{array}{l}\text { Mild } \\
\text { Mild }\end{array}$ & $\begin{array}{l}41 \\
51\end{array}$ & 1,644 & 2,398 & $\begin{array}{l}2,388 \\
1,000\end{array}$ & $\begin{array}{r}2,182 \\
904\end{array}$ & $2 \cdot \overline{2}$ & $=$ & $\overline{-}$ & $=$ & $0 . \overline{0}$ & $=$ & $=$ & $\bar{z}$ & $\begin{array}{l}0 \\
0\end{array}$ & $\begin{array}{l}0 \\
0\end{array}$ & & $\begin{array}{l}\text { Frequent V.E.s } \\
\text { S..B., N.R., } \\
\text { frequent V.E.s, } \\
\text { V.T. }\end{array}$ \\
\hline $\begin{array}{l}3 \\
4 \\
5\end{array}$ & $\begin{array}{l}\mathrm{H} \\
\mathrm{H} \\
\mathrm{H}\end{array}$ & $\begin{array}{l}47 \mathrm{M} . \\
47 \mathrm{M} \\
65 \mathrm{M}\end{array}$ & $\begin{array}{l}\mathbf{A} \\
\mathbf{A}\end{array}$ & $\begin{array}{l}\text { Mod. } \\
\text { Mod. } \\
\text { Mild }\end{array}$ & $\begin{array}{l}4 \\
21 \\
5\end{array}$ & $\begin{array}{l}1,667 \\
1,387\end{array}$ & $\begin{array}{l}2,222 \\
1,306 \\
1,510\end{array}$ & $\begin{array}{l}1,889 \\
1,306 \\
1,224\end{array}$ & $\begin{array}{l}1,629 \\
1,428 \\
694\end{array}$ & $\begin{array}{l}1.4 \\
4.5 \\
2 \cdot 3\end{array}$ & $\frac{1 \cdot 4}{1 \cdot 8}$ & $\frac{1 \cdot 6}{3 \cdot 3}$ & $\frac{2 \cdot 8}{14 \cdot 7}$ & $\begin{array}{l}0.4 \\
0.0 \\
0.5\end{array}$ & $\frac{7.5}{0.0}$ & $\frac{0.0}{0.0}$ & $\frac{0.0}{2.7}$ & $\begin{array}{r}0 \\
0 \\
157\end{array}$ & $\begin{array}{r}\mathbf{0} \\
\mathbf{0} \\
397\end{array}$ & & $\begin{array}{l}\text { None } \\
\text { V.F. } \\
\text { Frequent V.E.s }\end{array}$ \\
\hline $\begin{array}{l}6 \\
7 \\
8\end{array}$ & D & $\begin{array}{ll}58 & M . \\
56 & M . \\
58 & M .\end{array}$ & $\stackrel{A}{A}$ & $\begin{array}{l}\text { Mild } \\
\text { Mild } \\
\text { Mild }\end{array}$ & $\begin{array}{l}5 \\
4 \\
18\end{array}$ & $\begin{array}{l}634 \\
1,340 \\
571\end{array}$ & $\begin{array}{l}550 \\
1,380 \\
408\end{array}$ & $\begin{array}{l}560 \\
1,380 \\
612\end{array}$ & $\begin{array}{r}608 \\
1,370 \\
449\end{array}$ & $\begin{array}{l}1 \overline{8} \\
1.3\end{array}$ & $2 \cdot 9$ & $2 \cdot 0$ & $2 \cdot 25$ & $\begin{array}{l}0.3 \\
0.3\end{array}$ & $\underline{0.0}$ & 0.3 & 0.45 & $\begin{array}{l}1 \\
0 \\
0\end{array}$ & $\begin{array}{l}3 \\
0 \\
0\end{array}$ & & $\begin{array}{l}\text { V. bigeminy, v.T } \\
\text { Frrequent A.E.s } \\
\text { None }\end{array}$ \\
\hline $\begin{array}{r}8 \\
9 \\
10\end{array}$ & $\begin{array}{l}\mathrm{H} \\
\mathrm{H} \\
\mathrm{D}\end{array}$ & $\begin{array}{ll}58 & M . \\
58 & M \\
68 & M .\end{array}$ & $\hat{A}$ & $\begin{array}{l}\text { Mild } \\
\text { Mild } \\
\text { Mild }\end{array}$ & $\begin{array}{r}18 \\
33 \\
153\end{array}$ & $\begin{array}{r}800 \\
1,020 \\
1,960\end{array}$ & $\begin{array}{l}1,98 \\
1,914 \\
1,014\end{array}$ & $\begin{array}{l}1,12 \\
1,000 \\
1,060\end{array}$ & $\begin{array}{r}770 \\
7,018 \\
1,018\end{array}$ & $\begin{array}{l}1.05 \\
2.2 \\
1.05\end{array}$ & $\frac{2.1}{1.4}$ & $\frac{1 \cdot \overline{7}}{2 \cdot 1}$ & $\frac{2 \cdot 1}{2 \cdot 95}$ & $\begin{array}{l}0.25 \\
0.0 \\
0.0\end{array}$ & 0.05 & 0.0 & $0 . \overline{2}$ & $\begin{array}{r}177 \\
2 \\
8\end{array}$ & $\begin{array}{r}493 \\
8\end{array}$ & & $\begin{array}{l}\text { Frequent V.E.s } \\
\text { V.T. }\end{array}$ \\
\hline $\begin{array}{l}11 \\
12 \\
13\end{array}$ & $\begin{array}{l}\mathrm{H} \\
\mathrm{H}\end{array}$ & $\begin{array}{ll}61 & M . \\
55 & M \\
63 & M .\end{array}$ & A & $\begin{array}{l}\text { Mild } \\
\text { Mild } \\
\text { Mild }\end{array}$ & $\begin{array}{l}15 ! \\
15 \\
6 !\end{array}$ & $\begin{array}{r}8960 \\
1,100\end{array}$ & $\begin{array}{l}1,928 \\
1,880\end{array}$ & $\begin{array}{r}990 \\
1,460\end{array}$ & $\begin{array}{r}861 \\
916 \\
1,340\end{array}$ & $\begin{array}{l}1.05 \\
1.8 \\
1.4\end{array}$ & $\frac{1 \cdot 4}{1 \cdot 35}$ & $\frac{2 \cdot 1}{1 \cdot 5}$ & $\frac{2 \cdot 95}{1 \cdot 2}$ & $\begin{array}{l}0.0 \\
0.4 \\
0.0\end{array}$ & $\frac{0.1}{0.0}$ & $\frac{0.2}{0.0}$ & $\frac{0.3}{0.25}$ & $\begin{array}{l}8 \\
2 \\
0\end{array}$ & $\begin{array}{r}16 \\
7 \\
0\end{array}$ & & $\begin{array}{l}\text { None } \\
\text { Frequent V.E.s } \\
\text { None }\end{array}$ \\
\hline $\begin{array}{l}14 \\
15\end{array}$ & $\underset{\mathrm{H}}{\mathrm{D}}$ & $\begin{array}{l}50 \\
63 \\
{ }_{6}\end{array}$ & $\stackrel{\mathbf{A}}{\mathbf{P}}$ & $\begin{array}{l}\text { Mild } \\
\text { Mild }\end{array}$ & $\begin{array}{r}20 \\
2\end{array}$ & $\begin{array}{l}1,312 \\
730\end{array}$ & $\begin{array}{l}1,281 \\
1,793\end{array}$ & $\begin{array}{l}1,132 \\
1,524\end{array}$ & $\begin{array}{l}1,312 \\
1,095\end{array}$ & $\begin{array}{l}1 \cdot 2 \\
1 \cdot 8\end{array}$ & $\overline{3 \cdot 2}$ & $1 . \overline{55}$ & $\overline{2 \cdot 3}$ & $\begin{array}{l}0.0 \\
2.7\end{array}$ & $\overline{1 \cdot 8}$ & $\overline{1.45}$ & $0 . \overline{9}$ & 4 & 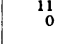 & N.R. 30 mins. & $\begin{array}{l}\text { None } \\
\text { Intermittent } \\
\text { L.B.B.B. }\end{array}$ \\
\hline $\begin{array}{l}16 \\
17 \\
18\end{array}$ & $\begin{array}{l}\mathrm{D} \\
\mathrm{H} \\
\mathrm{D}\end{array}$ & $\begin{array}{l}50 \mathrm{M} \\
73 \mathrm{M} \\
66 \mathrm{M}\end{array}$ & $\begin{array}{l}\mathrm{P} \\
\mathrm{P} \\
\mathrm{P}\end{array}$ & $\begin{array}{l}\text { Mild } \\
\text { Mod. } \\
\text { Mod. }\end{array}$ & $\begin{array}{l}2 \\
5 \\
5\end{array}$ & $\begin{array}{l}1,206 \\
1,281 \\
516\end{array}$ & $\begin{array}{r}1,428 \\
1,814 \\
936\end{array}$ & $\begin{array}{r}1,492 \\
1,389 \\
806\end{array}$ & $\begin{array}{l}1,238 \\
1,061 \\
902\end{array}$ & $\begin{array}{l}1.55 \\
2.2\end{array}$ & $1 \overline{8}$ & $\overline{1.1}$ & 1.5 & $\begin{array}{l}0.05 \\
0.3\end{array}$ & $\overline{0.25}$ & $\overline{0.75}$ & $0 . \overline{3}$ & $\begin{array}{l}0 \\
2 \\
2\end{array}$ & $\begin{array}{l}0 \\
2 \\
2\end{array}$ & 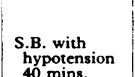 & $\begin{array}{l}\text { A.F., N.B., V.T. } \\
\text { Recurrent S.B. } \\
\text { Recurrent S.B., } \\
\text { I.V.R. }\end{array}$ \\
\hline $\begin{array}{l}19 \\
20\end{array}$ & $\stackrel{\mathrm{H}}{\mathrm{D}}$ & ${ }_{55}^{63} \mathrm{~F}$. & $\begin{array}{l}\mathbf{P} \\
\mathbf{P}\end{array}$ & $\begin{array}{l}\text { Mild } \\
\text { Mild }\end{array}$ & 9 & $\begin{array}{l}1,523 \\
1,666\end{array}$ & $\begin{array}{l}2,365 \\
1,334\end{array}$ & $\begin{array}{l}2,349 \\
1,416\end{array}$ & $\begin{array}{l}2,301 \\
1,374\end{array}$ & $\begin{array}{l}2.85 \\
1.95\end{array}$ & $5 \cdot 0$ & 1.75 & 3.3 & $\begin{array}{l}0.35 \\
0.0\end{array}$ & $\stackrel{0.0}{-}$ & 0.45 & $\stackrel{0.0}{-}$ & $\underset{2}{0}$ & $\begin{array}{l}0 \\
9\end{array}$ & & $\begin{array}{l}2^{6} \text { H.B. B. V.E.s, } \\
\text { Ron T V.E.s, }\end{array}$ \\
\hline$\frac{21}{22}$ & $\begin{array}{l}\mathbf{H} \\
\mathbf{D}\end{array}$ & $\begin{array}{l}46 \mathrm{M} \\
47 \mathrm{M}\end{array}$ & $\underset{\mathbf{P}}{\mathbf{P}}$ & $\begin{array}{l}\text { Mild } \\
\text { Mild }\end{array}$ & $\begin{array}{l}4 \\
2\end{array}$ & $\begin{array}{r}732 \\
1,107\end{array}$ & $\begin{array}{r}2,268 \\
964\end{array}$ & $\begin{array}{r}2,229 \\
964\end{array}$ & 2,058 & $\begin{array}{l}1.8 \\
2.5\end{array}$ & $\begin{array}{l}1.8 \\
0.8\end{array}$ & $\begin{array}{l}1 \cdot 7 \\
2 \cdot 3\end{array}$ & 1.9 & $\begin{array}{l}0.25 \\
0.65\end{array}$ & $\begin{array}{l}0.3 \\
0.5\end{array}$ & $\begin{array}{l}0.4 \\
0.35\end{array}$ & $\stackrel{0.1}{-}$ & -4 & 10 & $\begin{array}{l}\text { Frequent V.E.s } \\
30 \mathrm{mins}\end{array}$ & $\begin{array}{l}\text { I.V.R., V.T. } \\
\text { Frequent V.E.s }\end{array}$ \\
\hline $\begin{array}{l}23 \\
24\end{array}$ & $\begin{array}{l}\mathrm{H} \\
\mathrm{D}\end{array}$ & $\begin{array}{ll}50 & \mathrm{~F} . \\
63 & \mathrm{~F}\end{array}$ & A & $\begin{array}{l}\text { Mild } \\
\text { Mild }\end{array}$ & $4^{5 !}$ & $\begin{array}{l}1,120 \\
1,746\end{array}$ & $\begin{array}{l}1,880 \\
1,841\end{array}$ & $\begin{array}{l}1,480 \\
1,873\end{array}$ & $\begin{array}{l}1,420 \\
1,746\end{array}$ & $\begin{array}{l}2.8 \\
2.8\end{array}$ & 1.6 & 2.7 & 1.7 & $\begin{array}{l}0.0 \\
0.5\end{array}$ & 0.05 & 0.0 & 0.0 & $\frac{1}{12}$ & $\begin{array}{r}4 \\
35\end{array}$ & 30 mins. & 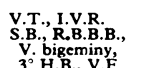 \\
\hline 25 & $\mathbf{H}$ & $66 \mathrm{M}$. & A & Mod. & 18 & 1,035 & 1,105 & 1,088 & 1,053 & - & - & - & - & - & - & - & - & 0 & 0 & & R.B.B.B., $3^{\circ}$ H.B., \\
\hline 26 & H & $53 \mathrm{M}$. & $\mathbf{P}$ & Mild & 3 & 1,698 & 2,236 & 2,156 & 1,679 & $2 \cdot 7$ & 6.5 & 1.8 & $2 \cdot 7$ & 0.05 & $1 \cdot 7$ & 0.4 & 0.5 & 4 & 9 & & S.B. with V.E.s \\
\hline
\end{tabular}

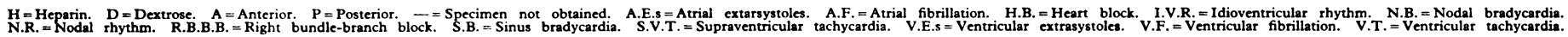

Miscellaneous: idioventricular rhythm, heart block, and bundlebranch block.

Twenty-six patients were initially investigated, but two remained unmatched at the end of the trial, both receiving heparin. They are included in the statistical analysis of the effect of heparin on F.F.A.s and plasma catecholamines and the predictive value of the initial F.F.A./catecholamine level for subsequent significant arrhythmias.

\section{Results}

Heparin 12,500 units consistently produced a rise in F.F.A. maximal in the sample taken 10 minutes after injection (Table I). The mean maximal increase was $66.8 \%$ (range 3$210 \%$ ) of the control value. There was no significant difference between the control F.F.A. levels and those obtained 60 minutes after heparin injection $(0 \cdot 1>P>0.05)$. The amount of dextrose given had no significant effect on F.F.A.s. At 10 minutes all the patients receiving heparin had F.F.A. levels greater than $1,000 \mu \mathrm{mole} / \mathrm{l}$., five being greater than 2,000 $\mu$ mole/l. Despite these high levels of F.F.A.s there was no significant increase in the number of ventricular ectopics during the hour following injection of heparin compared either with the 15-minute control period before injection or with the group receiving dextrose (Student's $t$ test). Case 15 developed transient nodal rhythm not requiring treatment 30 minutes after heparin injection. Case 18 had an episode of sinus bradycardia requiring intravenous atropine 40 minutes after dextrose injection. Case 22 developed multiple ventricular ectopics 30 minutes after dextrose injection which were treated with practolol (Eraldin). One patient from each group died. One (Case 3) died one week after admission with massive extension of his infarct, the other (Case 25) died following complete heart block 31 hours after admission.

Heparin did not have a consistent effect on plasma catecholamines, and the correlation coefficient ( $r$ ) between F.F.A.s and plasma catecholamines collected simultaneously did not achieve significance in any of the time groups.

Table II shows an analysis of arrhythmias occurring during the patients' stay in the coronary care unit related to the initial levels of F.F.A.s and catecholamines. A dividing line of $1,200 \mu \mathrm{mole} / 1$. was used for F.F.A.s and $1.5 \mu \mathrm{g}$./1. for plasma catecholamines. F.F.A. levels were not found to be a valuable predictive index for subsequent serious arrhythmias. Of 22 patients who had initial plasma catecholamine levels estimated 18 subsequently developed arrhythmias. The plasma noradrenaline level in all 18 was $>1.5 \mu \mathrm{g} . / 1$. All five
TABLE II

\begin{tabular}{|c|c|c|c|c|}
\hline & & & \multicolumn{2}{|c|}{ Control Serum F.F.A.s ( $\mu \mathrm{mole} / 1)}$. \\
\hline & & & $>1,200$ & $<1,200$ \\
\hline \multirow[t]{3}{*}{ 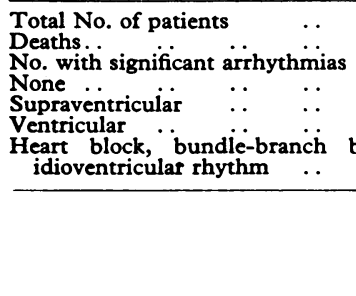 } & 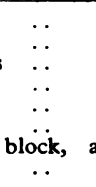 & $\begin{array}{r}\ldots \\
\ldots \\
\ldots \\
\ldots \\
\text { and } \\
\ldots\end{array}$ & $\begin{array}{r}11 \\
1 \\
9 \\
2 \\
6 \\
4 \\
3\end{array}$ & $\begin{array}{r}15 \\
1 \\
12 \\
3 \\
4 \\
8 \\
5\end{array}$ \\
\hline & & & \multicolumn{2}{|c|}{$\begin{array}{c}\text { Control Plasma Noradrenaline } \\
(\mu \mathrm{g} . / 1 .)\end{array}$} \\
\hline & & & $>1.5$ & $<1.5$ \\
\hline $\begin{array}{l}\text { Total No. of patients } \\
\text { No. with significant arrhythmias }\end{array}$ & $\cdots$ & $\because$ & 18 & $\begin{array}{l}5 \\
0\end{array}$ \\
\hline
\end{tabular}

patients with no arrhythmias had initial plasma noradrenaline levels $<1.5 \mu / 1$. This finding is highly significant $(P<0.01)$ and suggests that estimation of plasma noradrenaline in the early period following acute myocardial infarction may be of great value in predicting serious arrhythmias. Plasma adrenaline levels did not correlate with the subsequent development of arrhythmias.

\section{Discussion}

Most of the evidence available from clinical trials shows that after acute myocardial infarction short-term anticoagulation reduces the mortality and the incidence of thromboembolic complications (Douglas, 1962; Mitchell and Schwartz, 1965; Wright, 1968). Heparin is the preparation most commonly used to obtain an immediate anticoagulant effect and to maintain anticoagulation until the more slowly acting coumarin derivatives become effective. The recent suggestion that the elevation of F.F.A. levels by heparin may precipitate cardiac arrhythmias after acute myocardial infarction casts serious doubt on the safety of its use in this clinical situation (Kurien et al., 1969; Rutstein et al., 1969). This study indicates that heparin in therapeutic dosages has no significant arrhythmogenic properties.

There is lack of unanimity of opinion regarding the relative importance of raised F.F.A.s and plasma catecholamines in the genesis of cardiac dysrhythmias in patients with acute myocardial infarction. Both factors have been linked independently to arrhythmias following cardiac infarction in man (Oliver et al., 1968; Gupta et al., 1969; McDonald et al., 1969) and animals (Staszewska-Barczak and Ceremuźyński, 
1968; Ceremuzoyński et al., 1969; Kurien et al., 1969), and it is well known that catecholamines can release F.F.A.s from adipose tissue, thus raising their circulatory levels (Carlson, 1968).

The present study has shown that it is possible to predict those patients liable to develop significant arrhythmias following acute myocardial infarction by using a plasma noradrenaline level obtained early in the course of their illness. F.F.A. levels obtained simultaneously were of no value in predicting these vulnerable patients.

It is suggested that plasma noradrenaline estimations could be used to determine those patients who would benefit most from the continuous monitoring facilities of a coronary care unit.

If dysrhythmias following acute myocardial infarction are related to the excess circulating noradrenaline then clearly $\beta$ adrenergic blocking agents could be of prophylactic value. Propranolol has undergone several unsuccessful trials in this connexion (Clausen et al., 1966; Balcon et al., 1967; Norris et al., 1968). In these trials the dose administered was limited by the drug's negative inotropic properties. The more recently introduced practolol, which does not possess significant negative inotropic properties, might be of more value as a prophylactic measure in this clinical situation.

This study was suggested by Dr. J. F. Pantridge.
REFERENCES

Balcon, R., Jewitt, D. E., Davies, J. P. H., and Oram, S. (191,7). American Heart fournal, 74, 582 .

Carlson, L. A. (1968). In Acute Myocardial Infarction, ed. D. G. Julian and M. F. Oliver, p. 243. Edinburgh, Livingstone.

Ceremužnnski, L., Staszewska-Barczak, J., and Herbaczynska-Cedro, K. (1969). Cardiovascular Research, 3, 190.

Clausen, J., et al. (1966). Lancet, 2,920.

Douglas, A. S. (1962). Anticoagulant Therapy, p. 211. Oxford, Blackwell Scientific.

Gazes, P. C., Richardson, J. A., and Woods, E. F. (1959). Circulation, 19, 657.

Gupta, D. K., Young, R., Jewitt, D. E., Hartog, M., and Opie, L. H. (1969). Lancet, 2, 1209.

Kurien, V. A., and Oliver, M. F. (1966). Lancet, 2, 122.

Kurien, V. A., Yates, P. A., and Oliver, M. F. (1969). Lancet, 2, 185.

McCullough, H. (1968). Fournal of Clinical Pathology, 21, 759.

McDonald, L., Baker, C., Bray, C., McDonald, A., and Restieaux, N. (1969). Lancet, 2, 1021.

Maurer, B. J. (1970). "The Incidence of Venous Thrombosis following Acute Myocardial Infarction," paper presented to the Association of Physicians of Great Britain and Ireland on 11 April.

Mitchell, J. R. A., and Schwartz, C. J. (1965). Arterial Disease, p. 357. Oxford, Blackwell Scientific.

Mosinger, F. (1965). Fournal of Lipid Research, 6, 157

Norris, R. M., Caughey, D. E., and Scott, P. J. (1968). British Medical fournal, 2, 398.

Oliver, M. F., Kurien, V. A., and Greenwood, T. W. (1968). Lancet, 1, 710

Oliver, M. F., Kurien, V. A., and Greenwood, T. W. (1968). Lancer,
Richardson, J. A. (1963). Progress in Cardiovascular Disease, 6, 56.

Richardson, J. A. (1963). Progress in Cardiovascular Disease, 6, 56.
Rutenburg, H. L., Pamintuan, J. C., and Soloff, L. A. (1969). Lancet, 2, 559.

Rutenburg, H. L., and Soloff, L. A. (1970). Lancet, 1, 198.

Rutstein, D. D., Castelli, W. P., and Nickerson, R. J. (1969). Lancet, 1, 1003. Staszewska-Barczak, J., and Ceremuzyñski, L. (1968). Clinical Science, 34, 531 .

Trout, D. L., Estes, E. H., and Friedberg, S. J. (1960). Fournal of Lipid
Research, 1, 199.

Wright, I. S. (1968). South African Medical fournal, 42, 812.

\title{
Large-scale Digitoxin Intoxication
}

\author{
A. H. LELY,* M.D. ; C. H. J. VAN ENTER, ${ }^{*}$ M.D.
}

\begin{abstract}
Summary: Because of an error in the manufacture of digoxin tablets a large number of patients took tablets that contained $0.20 \mathrm{mg}$. of digitoxin and $0.05 \mathrm{mg}$. of digoxin instead of the prescribed $0.25 \mathrm{mg}$. of digoxin. The symptoms are described of 179 patients who took these tablets and suffered from digitalis intoxication. Of these patients, 125 had taken the faultily composed tablets for more than three weeks. In 48 patients 105 separate disturbances in rhythm or in atrioventricular conduction were observed on the electrocardiogram. Extreme fatigue and serious eye conditions were observed in $95 \%$ of the patients. Twelve patients had a transient psychosis. Extensive ophthalmological observations indicated that the visual complaints were most probably caused by a transient retrobulbar neuritis.
\end{abstract}

\section{Introduction}

At the beginning of February 1969 several patients were admitted to our hospital with symptoms of serious digitalis intoxication. They had all taken tablets containing $0.25 \mathrm{mg}$. of digoxin in normal daily dosage for months or years without incurring side-effects. This led us to suspect that the composition of the latest tablets supplied was faulty. On 7 February we asked the local pharmaceutical chemist to examine them. On 12 February we were informed that the tablets contained $0.20 \mathrm{mg}$. of digitoxin and $0.05 \mathrm{mg}$. of digoxin instead of $0.25 \mathrm{mg}$. of digoxin.

The chemist, who supplied drugs to the town of Veenendaal ( $\pm 30,000$ inhabitants) as well as to the local hospital had delivered the tablets on 6 December 1968. At the most, * Internist, Department of Internal Medicine, Juliana Hospital, Veenendaal,
The Netherlands. therefore, the patients had been taking them for 10 weeks. As we knew the dates of their delivery we could trace the number of days each patient had taken them.

Immediately it became known that digitoxin intoxication had occurred on a large scale many of the patients with serious symptoms were admitted to hospital. We went through the case histories of all those who had been admitted during the previous two months, and it became evident that we had in fact seen even more patients with digitalis intoxication.

In maintenance dosage digitoxin is weight for weight more active than digoxin. This is due to better resorption and slower excretion. Moe and Farah (1965) mentioned a daily maintenance dose of 0.05 to $0.2 \mathrm{mg}$. for digitoxin and 0.25 to $1 \mathrm{mg}$. for digoxin. Friedberg (1966) gives 0.05 to $0.2 \mathrm{mg}$. for digitoxin and 0.125 to $0.75 \mathrm{mg}$. for digoxin. According to these amounts, our patients had taken a daily dose of cardiac glycosides which was 2.2 to 4.2 times higher than that prescribed. Numerous publications have appeared since Withering's (1785) frequently quoted observations on digitalis in'oxication.

Fairly large studies of digitalis intoxication during digitalis theraoy among inpatients have been published-by Flaxman (1948) 30 cases, Crouch et al. (1956) 100 cases, Shrager (1957) 40 cases, von Capeller et al. (1959) 148 cases, Rodensky and Wasserman (1961) 88 cases, Dreifus et al. (1963) 161 cases, Schölmerich et al. (1964) 143 cases, Dubnow and Burchell (1965) 236 cascs, and Chung (1969) 180 cases. With the exception of the reports of Flaxman (1948) and Rodensky and Wasserman (1961) these cases were assembled in retrospect, with all the shortcomings of such situations. In addition, most of these series were based solely on the electrocardiographic evidence of digitalis intoxication. These drawbacks do not apply to the report by Church et al. (1962), who, in 41 episodes, deliberately gave excessive amounts of digitalis to 20 patients. 